\title{
A layered genetic algorithm with iterative diversification for optimization of flexible job shop scheduling problems
}

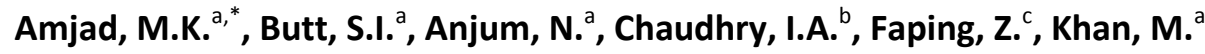 \\ ${ }^{a}$ School of Mechanical and Manufacturing Engineering, National University of Sciences and Technology, Islamabad, Pakistan \\ bepartment of Industrial Engineering, College of Engineering, University of Ha'il, Kingdom of Saudi Arabia

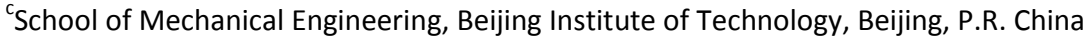

\begin{abstract}
A B S T R A C T
Flexible job shop scheduling problem (FJSSP) is a further expansion of the classical job shop scheduling problem (JSSP). FJSSP is known to be NP-hard with regards to optimization and hence poses a challenge in finding acceptable solutions. Genetic algorithm (GA) has successfully been applied in this regard since last two decades. This paper provides an insight into the actual complexity of selected benchmark problems through quantitative evaluation of the search space owing to their NP-hard nature. A four-layered genetic algorithm is then proposed and implemented with adaptive parameters of population initialization and operator probabilities to manage intensification and diversification intelligently. The concept of reinitialization is introduced whenever the algorithm is trapped in local minima till predefined number of generations. Results are then compared with various other standalone evolutionary algorithms for selected benchmark problems. It is found that the proposed GA finds better solutions with this technique as compared to solutions produced without this technique. Moreover, the technique helps to overcome the local minima trap. Further comparison and analysis indicate that the proposed algorithm produces comparative and improved solutions with respect to other analogous methodologies owing to the diversification technique.
\end{abstract}

\section{ARTICLE INFO}

Keywords:

Scheduling;

Flexible job shop scheduling problem (FJSSP);

Complexity;

Diversity;

Combinatorial optimization;

Genetic algorithm

*Corresponding author:

kamal.amjad@smme.edu.pk

(Amjad, M.K.)

Article history:

Received 19 May 2020

Revised 27 November 2020

Accepted 30 November 2020

\section{References}

[1] Pinedo, M.L. (2012). Scheduling: Theory, algorithms, and systems, Springer, Boston, USA, doi: 10.1007/978-14614-2361-4.

[2] Jain, A., Jain, P.K., Chan, F.T.S., Singh, S. (2013). A review on manufacturing flexibility, International Journal of Production Research, Vol. 51, No. 19, 5946-5970, doi: 10.1080/00207543.2013.824627.

[3] Dauzère-Péres, S., Lasserre, J.-B. (2012). An integrated approach in production planning and scheduling, SpringerVerlag, Berlin, Germany, doi: 10.1007/978-3-642-46804-9.

[4] Groover, M.P. (2015). Automation, production systems, and computer-integrated manufacturing, $4^{\text {th }}$ edition, Pearson Education, Delhi, India.

[5] Azzouz, A., Ennigrou, M., Said, L.B. (2017). A hybrid algorithm for flexible job-shop scheduling problem with setup times, International Journal of Production Management and Engineering, Vol. 5, No. 1, 23-30, doi: 10.4995/ijpme.2017.6618.

[6] Kacem, I. (2013). Genetic algorithms for solving flexible job shop scheduling problems, In: Jarboui, B., Siarry, P., Teghem, J. (eds.), Metaheuristics for Production Scheduling, John Wiley \& Sons, New York, USA, 19-44, doi: 10.1002/9781118731598.ch2.

[7] Chaudhry, I.A., Usman, M. (2017). Integrated process planning and scheduling using genetic algorithms, Tehnički Vjesnik - Technical Gazette, Vol. 24, No. 5, 1401-1409, doi: 10.17559/TV-20151121212910. 
[8] Cheng, T.C.E., Shafransky, Y., Ng, C.T. (2016). An alternative approach for proving the NP-hardness of optimization problems, European Journal of Operational Research, Vol. 248, No. 1, 52-58, doi: 10.1016/i.ejor. 2015.06.076.

[9] Brucker, P., Sotskov, Y.N., Werner, F. (2007). Complexity of shop-scheduling problems with fixed number of jobs: A survey, Mathematical Methods of Operations Research, Vol. 65, No. 3, 461-481, doi: 10.1007/s00186-006-0127$\underline{8}$.

[10] Candan, G., Yazgan, H.R. (2015). Genetic algorithm parameter optimisation using Taguchi method for a flexible manufacturing system scheduling problem, International Journal of Production Research, Vol. 53, No. 3, 897-915, doi: $10.1080 / 00207543.2014 .939244$.

[11] Ojstersek, R., Lalic, D., Buchmeister, B. (2019). A new method for mathematical and simulation modelling interactivity: A case study in flexible job shop scheduling, Advances in Production Engineering \& Management, Vol. 14, No. 4, 435-448, doi: 10.14743/apem2019.4.339.

[12] Borreguero-Sanchidrián, T., Pulido, R., García-Sánchez, Á., Ortega-Mier, M. (2018). Flexible job shop scheduling with operators in aeronautical manufacturing: A case study, IEEE Access, Vol. 6, 224-233, doi: 10.1109/ ACCESS.2017.2761994.

[13] Nidhiry, N.M., Saravanan, R. (2014). Scheduling optimization of a flexible manufacturing system using a modified NSGA-II algorithm, Advances in Production Engineering \& Management, Vol. 9, No. 3, 139-151, doi: 10.14743/ apem2014.3.183.

[14] Hecker, F.T., Hussein, W.B., Paquet-Durand, O., Hussein, M.A., Becker, T. (2013). A case study on using evolutionary algorithms to optimize bakery production planning, Expert Systems with Applications, Vol. 40, No. 17, 6837-6847, doi: 10.1016/j.eswa.2013.06.038.

[15] Amjad, M.K., Butt, S.I., Kousar, R., Ahmad, R., Agha, M.H., Faping, Z., Anjum, N., Asgher, U. (2018). Recent research trends in genetic algorithm based flexible job shop scheduling problems, Mathematical Problems in Engineering, Vol. 2018, No. 32, Article ID 9270802, doi: 10.1155/2018/9270802.

[16] Zhang, J., Ding, G., Zou, Y., Qin, S., Fu, J. (2019). Review of job shop scheduling research and its new perspectives under Industry 4.0, Journal of Intelligent Manufacturing, Vol. 30, No. 4, 1809-1830, doi: 10.1007/s10845-0171350-2.

[17] Janes, G., Perinic, M., Jurkovic, Z. (2017). An efficient genetic algorithm for job shop scheduling problems, Tehnički Vjesnik - Technical Gazette, Vol. 24, No. 4, 1243-1247, doi: 10.17559/TV-20150527133957.

[18] Chaudhry, I.A., Khan, A.A. (2016). A research survey: Review of flexible job shop scheduling techniques, International Transactions in Operational Research, Vol. 23, No. 3, 551-591, doi: 10.1111/itor12199.

[19] Çaliș, B., Bulkan, S. (2015). A research survey: Review of AI solution strategies of job shop scheduling problem, Journal of Intelligent Manufacturing, Vol. 26, No. 5, 961-973, doi: 10.1007/s10845-013-0837-8.

[20] Ida, K., Oka, K. (2011). Flexible job-shop scheduling problem by genetic algorithm, Electrical Engineering in Japan, Vol. 177, No. 3, 28-35, doi: 10.1002/eej.21194.

[21] Kacem, I., Hammadi, S., Borne, P. (2002). Approach by localization and multiobjective evolutionary optimization for flexible job-shop scheduling problems, IEEE Transactions on Systems, Man, and Cybernetics, Part C (Applications and Reviews), Vol. 32, No. 1,1-13, doi: 10.1109/TSMCC.2002.1009117.

[22] Gao, J., Gen, M., Sun, L., Zhao, X. (2007). A hybrid of genetic algorithm and bottleneck shifting for multiobjective flexible job shop scheduling problems, Computers \& Industrial Engineering, Vol. 53, No. 1, 149-162, doi: 10.1016/i.cie.2007.04.010.

[23] Pezzella, F., Morganti, G., Ciaschetti, G. (2008). A genetic algorithm for the flexible job-shop scheduling problem, Computers \& Operations Research, Vol. 35, No. 10, 3202-3212, doi: 10.1016/i.cor.2007.02.014.

[24] Gu, X., Huang, M., Liang, X. (2019). An improved genetic algorithm with adaptive variable neighborhood search for FJSP, Algorithms, Vol. 12, No. 11, Article No. 243, doi: 10.3390/a12110243.

[25] Wang, L., Luo, C., Cai, J. (2017). A variable interval rescheduling strategy for dynamic flexible job shop scheduling problem by improved genetic algorithm, Journal of Advanced Transportation, Vol. 2017, Article ID 1527858, doi: $10.1155 / 2017 / 1527858$.

[26] Xiong, J., Tan, X., Yang, K.-W., Xing, L.-N., Chen, Y.-W. (2012). A hybrid multiobjective evolutionary approach for flexible job-shop scheduling problems, Mathematical Problems in Engineering, Vol. 2012, Article ID 478981, doi: $10.1155 / 2012 / 478981$.

[27] Teekeng, W., Thammano, A. (2012). Modified genetic algorithm for flexible job-shop scheduling problems, Procedia Computer Science, Vol. 12, 122-128, doi: 10.1016/i.procs.2012.09.041.

[28] Framinan, J.M., Leisten, R., García, R.R. (2014). Manufacturing scheduling systems, Springer-Verlag, London, United Kingdom, doi: 10.1007/978-1-4471-6272-8.

[29] Zhang, G., Gao, L., Shi, Y. (2011). An effective genetic algorithm for the flexible job-shop scheduling problem, Expert Systems with Applications, Vol. 38, No. 4, 3563-3573, doi: 10.1016/i.eswa.2010.08.145.

[30] Fattahi, P., Saidi Mehrabad, M., Jolai, F. (2007). Mathematical modeling and heuristic approaches to flexible job shop scheduling problems, Journal of Intelligent Manufacturing, Vol. 18, No. 3, 331-342, doi: 10.1007/s10845007-0026-8.

[31] Song, W.J., Zhang, C.Y., Lin, W.W., Shao, X.Y. (2014). Flexible job-shop scheduling problem with maintenance activities considering energy consumption, Applied Mechanics and Materials, Vol. 521, 707-713, doi: 10.4028/www.scientific.net/AMM.521.707.

[32] De Giovanni, L., Pezzella, F. (2010). An improved genetic algorithm for the distributed and flexible job-shop scheduling problem, European Journal of Operational Research, Vol. 200, No. 2, 395-408, doi: 10.1016/ j.ejor.2009.01.008. 
[33] Nouri, H.E., Driss, O.B., Ghédira, K. (2017). Solving the flexible job shop problem by hybrid metaheuristics-based multiagent model, Journal of Industrial Engineering International, Vol. 14, 1-14, doi: 10.1007/s40092-017-0204z.

[34] Zandieh, M., Mahdavi, I., Bagheri, A. (2008). Solving the flexible job-shop scheduling problem by a genetic algorithm, Journal of Applied Sciences, Vol. 8, No. 24, 4650-4655, doi: 10.3923/jas.2008.4650.4655.

[35] Bagheri, A., Zandieh, M., Mahdavi, I., Yazdani, M. (2010). An artificial immune algorithm for the flexible job-shop scheduling problem, Future Generation Computer Systems, Vol. 26, No. 4, 533-541, doi: 10.1016/i.future. 2009.10.004.

[36] Behnke, D., Geiger, M.J. (2012). Test instances for the flexible job shop scheduling problem with work centers, Universitätsbibliothek der Helmut-Schmidt-Universität, Hamburg, Germany, from https://d-nb.info/1023241773/34, accessed May 2020. 


\section{APEM}

\title{
Večplastni genetski algoritem s ponavljalno diverzifikacijo za optimizacijo problema fleksibilnega terminiranja proizvodnje po naročilu
}

\author{
Amjad, M.K. ${ }^{a}{ }^{*}$, Butt, S.I. ${ }^{a}$, Anjum, N. ${ }^{a}$, Chaudhry, I.A. ${ }^{b}$, Faping, Z. ${ }^{c}$, Khan, M. ${ }^{a}$ \\ aSchool of Mechanical and Manufacturing Engineering, National University of Sciences and Technology, Islamabad, Pakistan \\ bepartment of Industrial Engineering, College of Engineering, University of Ha'il, Kingdom of Saudi Arabia \\ 'School of Mechanical Engineering, Beijing Institute of Technology, Beijing, P.R. China
}

\section{POVZETEK}

Problem prilagodljivega terminiranja proizvodnje po naročilu (FJSSP) je razširitev klasičnega problema terminiranja proizvodnje po naročilu (JSSP). Znano je, da je FJSSP NP-težki optimizacijski problem, kar predstavlja glavni izziv pri iskanju sprejemljivih rešitev. Genetski algoritem (GA) se za reševanje takšnih problemov uporablja že zadnji dve desetletji. Ta prispevek obravnava kompleksnost izbranih standardnih testnih primerov s kvantitativnim vrednotenjem prostora rešitev, ki je vzrok njihove NP-težke narave. Za inteligentno upravljanje intenziviranja in diverzifikacije je predlagan in uporabljen štiriplastni genetski algoritem s prilagodljivimi parametri za inicializacijo populacije in prilagodljivimi verjetnostmi operatorjev GA. Koncept ponovne inicializacije se uvede vsakič, ko se algoritem ujame v lokalni minimum, preden doseže vnaprej določeno število generacij. Rezultate, dobljene s predlaganim algoritmom za standardne testne primere, smo primerjani z rezultati, dobljenimi z drugimi evolucijskimi algoritmi. Ugotovljeno je bilo, da predlagani GA z iterativno diverzifikacijo najde boljše rešitve v primerjavi z rešitvami, pridobljenimi brez predlaganega pristopa. Poleg tega ta pristop pripomore, da se reševanje ne konča $v$ lokalnem minimumu. Nadaljnja analiza in primerjava $s$ konkurenčnimi metodologijami kažeta, da predlagani algoritem, zaradi tehnike diverzifikacije, najde primerljive in boljše rešitve.

\section{PODATKI O ČLANKU}

Ključne besede:

Terminiranje;

Prilagodljivo terminiranje proizvodnje po naročilu (FJSSP);

Kompleksnost;

Raznolikost;

Kombinatorna optimizacija;

Genetski algoritem

*Kontaktna oseba: kamal.amjad@smme.edu.pk (Amjad, M.K.)

Zgodovina članka:

Prejet 19. maja 2020

Popravljen 27. novembra 2020

Sprejet 30. novembra 2020 